\title{
Some problems with reproducing the Standard Model fields and interactions in five-dimensional warped brane world models
}

\author{
Mikhail N. Smolyakov, Igor P. Volobuev \\ Skobeltsyn Institute of Nuclear Physics, Lomonosov Moscow State University, \\ 119991, Moscow, Russia
}

\begin{abstract}
In the present paper we examine, from the purely theoretical point of view and in a model-independent way, the case, when matter, gauge and Higgs fields are allowed to propagate in the bulk of five-dimensional brane world models with compact extra dimension, and the Standard Model fields and their interactions are supposed to be reproduced by the corresponding zero Kaluza-Klein modes. An unexpected result is that in order to avoid possible pathological behavior in the fermion sector, it is necessary to impose constraints on the fermion field Lagrangian. In the case when the fermion zero modes are supposed to be localized at one of the branes, these constraints imply an additional relation between the vacuum profile of the Higgs field and the form of the background metric. Moreover, this relation between the vacuum profile of the Higgs field and the form of the background metric results in the exact reproduction of the gauge boson and fermion sectors of the Standard Model by the corresponding zero mode four-dimensional effective theory in all the physically relevant cases, allowed by the absence of pathologies. Meanwhile, deviations from these conditions can lead either back to pathological behavior in the fermion sector or to a variance between the resulting zero mode four-dimensional effective theory and the Standard Model, which, depending on the model at hand, may, in principle, result in constraints putting the theory out of the reach of the present day experiments.
\end{abstract}

\section{Introduction}

Models with extra dimensions have been attracting a great interest during the last fifteen years. There were many attempts to solve various theoretical problems with the help of extra dimensions. A wide branch of multidimensional models is that of brane world models, which were proposed in their modern form in [1, 2. Although some theoretical problems (such as, for example, the hierarchy problem of gravitational interaction) were successfully solved within the framework of brane world models, realistic theories must also describe all the physical aspects of our four-dimensional world. In particular, they must correctly reproduce the interactions of the Standard Model (SM) particles that have already been tested experimentally.

In the original formulation of brane world models the SM fields were supposed to be located on a brane (in the Randall-Sundrum model [2], on the TeV brane). Later the idea of brane worlds was joined with the idea that all the fields can propagate in extra dimensions [3] thus giving rise to the theories with universal extra dimensions, where the matter, gauge and Higgs 
fields are allowed to propagate in the bulk of five-dimensional brane world models with compact extra dimension. In this case all these fields posses towers of Kaluza-Klein excitations, their zero modes being the SM fields. There exist many papers describing how the SM can be embedded this way into multidimensional brane worlds and what new effects can be produced in such theories.

However, there are some finer points that have been missed in the previous studies. Below we will discuss them in detail from a purely theoretical point of view and in a mathematically consistent way. In particular, we argue that it is impossible to exactly reproduce by the zero Kaluza-Klein modes the electroweak gauge boson sector of the SM in the effective four-dimensional theory, unless the vacuum profile of the Higgs field in the extra dimension behaves like the square root of the inverse warp factor. The same vacuum profile of the Higgs field (together with extra constraints on the parameters of the five-dimensional fermion field Lagrangian) is necessary for reproducing the fermion sector of the SM. An unexpected result is that deviations from these conditions may lead either to pathologies in the fermion sector when expanding in Kaluza-Klein modes (for example, in the case admitting consistent localization of the fermion zero mode at one of the branes) or to an additional modification of the couplings of fermions to gauge bosons in the zero mode sector, which may lead, in principle, to severe restrictions on the value of the five-dimensional energy scale (like the constraints coming from the zero mode gauge boson sector [4, 5]). Meanwhile, the demand for the extra restriction on the vacuum profile of the Higgs field, mentioned above, leads to some difficulties, such as the necessity for an extra fine-tuning. This problem needs to be addressed, at least for better understanding the structure of brane world models.

The paper is organized as follows. In Section 2 we consider an illustrative example of bulk gauge fields interacting with the bulk Higgs scalar field and show which conditions should be fulfilled in order the gauge fields exactly reproduce the electroweak gauge sector of the SM by the lowest Kaluza-Klein modes. In Section 3 fermions are examined in the same way. In Section 4 we consider interactions between fermions and gauge bosons in a simple theory, where pathologies in the fermion sector are absent, whereas no extra conditions on the Higgs field are imposed. The obtained results are discussed in the last section.

\section{Gauge fields}

Let us take a five-dimensional space-time with the coordinates $x^{M}=\left\{x^{\mu}, z\right\}, M=0,1,2,3,5$. The compact extra dimension is supposed to form the orbifold $S^{1} / Z_{2}$, which can be represented as the circle with the coordinate $-L \leq z \leq L$ and the points $-z$ and $z$ identified. In what follows, we will use the notation $x$ for the coordinates $x^{\mu}$. We consider the following standard form of the background metric, which is often used in brane world models:

$$
d s^{2}=e^{2 \sigma(z)} \eta_{\mu \nu} d x^{\mu} d x^{\nu}-d z^{2}
$$

This metric is assumed to correspond to a regular brane world model, i.e. it is a solution to equations of motion for five-dimensional gravity, two branes with tension and, for example, a stabilizing bulk scalar field. We do not specify the explicit form of the solution for $\sigma(z)$. 
We start with the gauge fields and choose the following action of an $S U(2) \times U(1)$ gauge invariant model in this background:

$$
S=\int d^{4} x d z \sqrt{g}\left(-\frac{\xi^{2}}{4} F^{a, M N} F_{M N}^{a}-\frac{\xi^{2}}{4} B^{M N} B_{M N}+g^{M N}\left(D_{M} H\right)^{\dagger} D_{N} H-V\left(H^{\dagger} H\right)\right),
$$

where

$$
\begin{aligned}
& F_{M N}^{a}=\partial_{M} A_{N}^{a}-\partial_{N} A_{M}^{a}+g \epsilon^{a b c} A_{M}^{b} A_{N}^{c}, \\
& B_{M N}=\partial_{M} B_{N}-\partial_{N} B_{M}, \\
& D_{M} H=\left(\partial_{M}-i g \frac{\tau^{a}}{2} A_{M}^{a}-i \frac{g^{\prime}}{2} B_{M}\right) H
\end{aligned}
$$

and the fields satisfy the orbifold symmetry conditions $A_{\mu}^{a}(x,-z)=A_{\mu}^{a}(x, z), A_{5}^{a}(x,-z)=$ $-A_{5}^{a}(x, z), B_{\mu}(x,-z)=B_{\mu}(x, z), B_{5}(x,-z)=-B_{5}(x, z), H(x,-z)=H(x, z)$. Here $\xi=\frac{1}{\sqrt{2 L}}$ is a constant, which is introduced for convenience and chosen so that the dimension of the bulk gauge fields is mass. The scalar field potential can include brane-localized terms of the form $\lambda_{1}\left(H^{\dagger} H\right) \delta(z)$ and $\lambda_{2}\left(H^{\dagger} H\right) \delta(z-L)$. It is easy to see that action (2), which has a rather standard form, resembles the bosonic sector of the electroweak part of the ordinary four-dimensional SM.

This action gives rise to the equations of motion for the gauge and the Higgs fields that look like

$$
\begin{aligned}
& \nabla_{N} F^{a, M N}+g \epsilon^{a b c} A_{N}^{b} F^{c, M N}+i \frac{g}{\xi^{2}}\left(\left(D^{M} H\right)^{\dagger} \frac{\tau^{a}}{2} H-H^{\dagger} \frac{\tau^{a}}{2} D^{M} H\right)=0 \\
& \nabla_{N} B^{M N}+i \frac{g^{\prime}}{2 \xi^{2}}\left(\left(D^{M} H\right)^{\dagger} H-H^{\dagger} D^{M} H\right)=0 \\
& \nabla^{M} D_{M} H-g^{M N}\left(i g \frac{\tau^{a}}{2} A_{M}^{a}+i \frac{g^{\prime}}{2} B_{M}\right) D_{N} H+\frac{d V}{d\left(H^{\dagger} H\right)} H=0
\end{aligned}
$$

$\nabla^{M}$ denoting the covariant derivative with respect to metric (1).

Let us consider the vacuum solution for these fields. The vacuum solution, breaking the gauge group $S U(2) \times U(1)$ to $U(1)_{e m}$, leaving the Poincare invariance in four-dimensional space-time intact and satisfying equations (6), (7), can be taken in the form

$$
A_{M}^{a} \equiv 0, \quad B_{M} \equiv 0, \quad H_{0} \equiv\left(\begin{array}{c}
0 \\
\frac{v(z)}{\sqrt{2}}
\end{array}\right)
$$

where $v(z)$ is a real function. It is not difficult to understand that in the general case the vacuum solution for the Higgs field $v(z)$ may depend on the coordinate of the extra dimension. Of course, the scalar field potential must provide for such a solution to equation (8). At this point we do not specify the explicit form of $v(z)$.

Now let us turn to examining the excitations in the model at hand. Below we will be interested in the behavior of only the four-vector components of the five-dimensional gauge fields, whose zero modes must play the role of the SM gauge bosons. For this reason, from here on we retain only these components of the gauge fields and drop the components $A_{5}^{a}, B_{5}$ 
of the vector fields and the fluctuations of the Higgs field. From action (21) it is easy to get the following effective action for the four-vector components of the five-dimensional gauge fields:

$$
\begin{gathered}
S_{\text {eff }}=\int d^{4} x d z\left(-\frac{\xi^{2}}{4} \eta^{\mu \nu} \eta^{\alpha \beta} F_{\mu \alpha}^{a} F_{\nu \beta}^{a}+e^{2 \sigma} \frac{\xi^{2}}{2} \eta^{\mu \nu} \partial_{5} A_{\mu}^{a} \partial_{5} A_{\nu}^{a}-\frac{\xi^{2}}{4} \eta^{\mu \nu} \eta^{\alpha \beta} B_{\mu \alpha} B_{\nu \beta}\right. \\
\left.+e^{2 \sigma} \frac{\xi^{2}}{2} \eta^{\mu \nu} \partial_{5} B_{\mu} \partial_{5} B_{\nu}+e^{2 \sigma} \eta^{\mu \nu} H_{0}^{\dagger}\left(g \frac{\tau^{a}}{2} A_{\mu}^{a}+\frac{g^{\prime}}{2} B_{\mu}\right)\left(g \frac{\tau^{a}}{2} A_{\nu}^{a}+\frac{g^{\prime}}{2} B_{\nu}\right) H_{0}\right),
\end{gathered}
$$

where we have also dropped the terms containing only the vacuum configuration of the Higgs field.

Now we are ready to perform the Kaluza-Klein mode decomposition. First, using the standard redefinition

$$
Z_{\mu}=\frac{1}{\sqrt{g^{2}+g^{\prime 2}}}\left(g A_{\mu}^{3}-g^{\prime} B_{\mu}\right), A_{\mu}=\frac{1}{\sqrt{g^{2}+g^{\prime 2}}}\left(g B_{\mu}+g^{\prime} A_{\mu}^{3}\right), W_{\mu}^{ \pm}=\frac{1}{\sqrt{2}}\left(A_{\mu}^{1} \mp i A_{\mu}^{2}\right)
$$

we can pass to the physical degrees of freedom of the theory. Next, let us consider only the quadratic part of effective action (10) in terms of these new fields. It takes the form

$$
\begin{array}{r}
S_{e f f}=\int d^{4} x d z\left(-\frac{\xi^{2}}{2} \eta^{\mu \nu} \eta^{\alpha \beta} W_{\mu \alpha}^{+} W_{\nu \beta}^{-}-\frac{\xi^{2}}{4} \eta^{\mu \nu} \eta^{\alpha \beta} F_{\mu \alpha} F_{\nu \beta}-\frac{\xi^{2}}{4} \eta^{\mu \nu} \eta^{\alpha \beta} Z_{\mu \alpha} Z_{\nu \beta}\right. \\
+e^{2 \sigma} \xi^{2} \eta^{\mu \nu} \partial_{5} W_{\mu}^{+} \partial_{5} W_{\nu}^{-}+e^{2 \sigma} \frac{\xi^{2}}{2} \eta^{\mu \nu} \partial_{5} A_{\mu} \partial_{5} A_{\nu}+e^{2 \sigma} \frac{\xi^{2}}{2} \eta^{\mu \nu} \partial_{5} Z_{\mu} \partial_{5} Z_{\nu} \\
\left.+e^{2 \sigma} v^{2}(z) \frac{g^{2}}{4} \eta^{\mu \nu} W_{\mu}^{+} W_{\nu}^{-}+e^{2 \sigma} v^{2}(z) \frac{g^{2}+g^{\prime 2}}{8} \eta^{\mu \nu} Z_{\mu} Z_{\nu}\right)
\end{array}
$$

where $W_{\mu \nu}^{ \pm}=\partial_{\mu} W_{\nu}^{ \pm}-\partial_{\nu} W_{\mu}^{ \pm}, F_{\mu \nu}=\partial_{\mu} A_{\nu}-\partial_{\nu} A_{\mu}, Z_{\mu \nu}=\partial_{\mu} Z_{\nu}-\partial_{\nu} Z_{\mu}$. The equations for the wave functions and the masses of the Kaluza-Klein modes are

$$
\begin{aligned}
-m_{W, n}^{2} f_{W, n}-\partial_{5}\left(e^{2 \sigma} \partial_{5} f_{W, n}\right)+\frac{g^{2}}{4 \xi^{2}} e^{2 \sigma} v^{2}(z) f_{W, n} & =0, \\
-m_{Z, n}^{2} f_{Z, n}-\partial_{5}\left(e^{2 \sigma} \partial_{5} f_{Z, n}\right)+\frac{g^{2}+g^{\prime 2}}{4 \xi^{2}} e^{2 \sigma} v^{2}(z) f_{Z, n} & =0, \\
-m_{A, n}^{2} f_{A, n}-\partial_{5}\left(e^{2 \sigma} \partial_{5} f_{A, n}\right) & =0 .
\end{aligned}
$$

where the subscript $n$ denotes the number of the corresponding Kaluza-Klein mode. As usual, the lowest (zero) Kaluza-Klein modes of the fields are supposed to correspond to the fourdimensional SM particles. So, below we will focus only on the zero modes.

It follows from (15) that the solution for the lowest mode of the field $A_{\mu}$ (the photon) is $m_{A, 0}=0$ and $f_{A, 0}(z) \equiv$ const, i.e. its wave function does not depend on the coordinate of the extra dimension. This is an important result, which provides the universality of the electromagnetic charge [6]. But, as one sees from (13) and (14), in the general case it is not so for the zero modes of the fields $W_{\mu}$ and $Z_{\mu}$, which correspond to the SM massive gauge bosons. The latter has the following well-known consequences. Indeed, in the SM the selfcoupling of massive gauge bosons comes from the term $F^{a, \mu \nu} F_{\mu \nu}^{a}$ and the corresponding coupling constants are defined only by the structure of the gauge group. In the five-dimensional case under consideration the self-coupling terms also come from the same term of (10), but now the 
corresponding coupling constants are also defined by the overlap integrals over the coordinate $z$, which include the wave functions $f_{W, 0}(z)$ and $f_{Z, 0}(z)$. The only case, when the zero mode sector of the model automatically completely coincides with the electroweak gauge boson sector of the $\mathrm{SM}$, is the one, where the wave functions $f_{W, 0}(z)$ and $f_{Z, 0}(z)$ do not depend on the coordinate of the extra dimension. In this case the self-coupling constants of the massive gauge bosons are defined in terms of the constants $g$ and $g^{\prime}$ exactly in the same way as in the ordinary SM. The independence of the wave functions $f_{W, 0}(z)$ and $f_{Z, 0}(z)$ on the coordinate of the extra dimension can be achieved only when $e^{2 \sigma} v^{2}(z) \equiv$ const, i.e., when

$$
v(z) \equiv \xi \tilde{v} e^{-\sigma},
$$

where $\tilde{v}$ is a constant of dimension $M$. For the choice (16), the masses of the zero mode gauge bosons are given by

$$
m_{W, 0}=\frac{g \tilde{v}}{2}, \quad m_{Z, 0}=\frac{\sqrt{g^{2}+g^{\prime 2}} \tilde{v}}{2} .
$$

Thus, in the case under consideration $\tilde{v}$ must coincide with the Higgs field vacuum expectation value of the SM.

Of course, the results presented above are rather trivial. Moreover, it is well known that in the general case a modification of the shapes of the zero mode gauge boson wave functions has an influence on the electroweak observables, this problem was discussed in detail in [4, 5]. It is shown in these papers that, for example, in the case of the Randall-Sundrum model [2] such a modification leads to restrictions on the value of the five-dimensional energy scale, which put the theory out of the reach of the present day experiments. For the choice (16) the lowest mode four-dimensional effective theory exactly reproduces the electroweak gauge boson sector of the SM, thus imposing no restrictions on the value of the five-dimensional energy scale. Meanwhile, one can imagine that there exists a profile for the Higgs vacuum solution, which differs from (16) but provides somehow the necessary values of the zero mode gauge boson masses and selfcoupling constants with a good accuracy. Unfortunately, the situation becomes more involved, when one comes to fermions.

\section{Fermions}

It is well known that, since there is no chirality in five-dimensional space-time, in order to obtain a nonzero mass term for the zero Kaluza-Klein fermion mode via the Higgs mechanism it is necessary to take two five-dimensional spinor fields (see, for example, [7, 8, 9]) satisfying the orbifold symmetry conditions

$$
\begin{array}{r}
\Psi_{1}(x,-z)=\gamma^{5} \Psi_{1}(x, z), \\
\Psi_{2}(x,-z)=-\gamma^{5} \Psi_{2}(x, z) .
\end{array}
$$

Thus, as a simple example, we consider a model with the action of the most general form

$$
\begin{aligned}
S & =\int d^{4} x d z \sqrt{g}\left(E_{N}^{M} i \bar{\Psi}_{1} \Gamma^{N} \nabla_{M} \Psi_{1}+E_{N}^{M} i \bar{\Psi}_{2} \Gamma^{N} \nabla_{M} \Psi_{2}\right. \\
& \left.-F_{1}(z) \bar{\Psi}_{1} \Psi_{1}-F_{2}(z) \bar{\Psi}_{2} \Psi_{2}-G(z)\left(\bar{\Psi}_{2} \Psi_{1}+\bar{\Psi}_{1} \Psi_{2}\right)\right),
\end{aligned}
$$


where $M, N=0,1,2,3,5, \Gamma^{\mu}=\gamma^{\mu}, \Gamma^{5}=i \gamma^{5}, \nabla_{M}$ is the covariant derivative containing the spin connection, $E_{N}^{M}$ is the vielbein, $F_{1,2}(z)$ and $G(z)$ are some functions satisfying the symmetry conditions $F_{1,2}(-z)=-F_{1,2}(z)$ and $G(-z)=G(z)$. For the case of metric (11) action (20) can be rewritten in the form (see, for example, [9, 10] for the explicit form of the vielbein and spin connections)

$$
\begin{array}{r}
S=\int d^{4} x d z e^{4 \sigma}\left(e^{-\sigma} i \bar{\Psi}_{1} \gamma^{\mu} \partial_{\mu} \Psi_{1}-\bar{\Psi}_{1} \gamma^{5}\left(\partial_{5}+2 \sigma^{\prime}\right) \Psi_{1}-F_{1}(z) \bar{\Psi}_{1} \Psi_{1}\right. \\
\left.+e^{-\sigma} i \bar{\Psi}_{2} \gamma^{\mu} \partial_{\mu} \Psi_{2}-\bar{\Psi}_{2} \gamma^{5}\left(\partial_{5}+2 \sigma^{\prime}\right) \Psi_{2}-F_{2}(z) \bar{\Psi}_{2} \Psi_{2}-G(z)\left(\bar{\Psi}_{2} \Psi_{1}+\bar{\Psi}_{1} \Psi_{2}\right)\right),
\end{array}
$$

where $^{\prime}=\partial_{5}$. The equations of motion, following from this action, take the form

$$
\begin{aligned}
& e^{-\sigma} i \gamma^{\mu} \partial_{\mu} \Psi_{1}-\gamma^{5}\left(\partial_{5}+2 \sigma^{\prime}\right) \Psi_{1}-F_{1}(z) \Psi_{1}-G(z) \Psi_{2}=0 \\
& e^{-\sigma} i \gamma^{\mu} \partial_{\mu} \Psi_{2}-\gamma^{5}\left(\partial_{5}+2 \sigma^{\prime}\right) \Psi_{2}-F_{2}(z) \Psi_{2}-G(z) \Psi_{1}=0
\end{aligned}
$$

Suppose that $G(z) \equiv 0$. In this case there always exists the solution

$$
\Psi_{1}=C_{f} \exp \left[-\int_{0}^{z} F_{1}(y) d y-2 \sigma(z)\right] \psi_{L}(x), \quad i \gamma^{\mu} \partial_{\mu} \psi_{L}=0, \quad \gamma^{5} \psi_{L}=\psi_{L}
$$

where $C_{f}$ is a normalization constant, describing a massless four-dimensional fermion. An analogous solution exists for the field $\Psi_{2}$ (but with a right-handed four-dimensional fermion). The latter clearly indicates that the existence of only one five-dimensional fermion is not enough to provide a massive four-dimensional lowest mode. This also indicates that it is the term with $G(z) \not \equiv 0$ that is responsible for the generation of the masses of the zero mode fermions. Thus, the function $G(z)$ should be somehow connected with the five-dimensional Higgs field. It is natural to take this function as

$$
G(z) \equiv h v(z)
$$

where $h$ is a coupling constant of dimension $M^{-\frac{1}{2}}$. Such a construction may arise, when one considers the standard Higgs mechanism in the bulk after the spontaneous symmetry breaking, leading to (9), whereas the zero modes of the fields $\Psi_{1}, \Psi_{2}$ are supposed to represent a massive lepton (for example, the electron).

It should be noted that the "localizing" functions $F_{1}(z)$ and $F_{2}(z)$ are not connected with the Higgs field in the general case. Meanwhile, the corresponding terms are not forbidden and, according to (24), they are responsible for the localization of the lowest fermion Kaluza-Klein modes. In fact, the form of the terms with the functions $F_{1}(z)$ and $F_{2}(z)$ in (20) is the only one suitable for the localization of the fermion zero modes in a consistent field-theoretical manner, though the origin of the localizing functions can be different. In the context of multidimensional models, such a mechanism was proposed in [11] for the theory with one infinite extra dimension, in which the localizing function was just a profile of the topological soliton modeling a domain wall. An analogous mechanism (but with another form of the localizing function) was used in [12, where fermion fields were also supposed to be confined to a thick domain wall in flat extra dimensions, but different fermions were localized at different points of the wall. In fivedimensional brane world models the localizing functions are usually not explicitly connected 
with something like a domain wall, very often the corresponding terms have the form similar to the standard fermion mass term, but with an antisymmetric "mass" according to the orbifold symmetry, i.e., $F(z)=C \operatorname{sign}(z)$. The value of the constant $C$ defines at which brane the fermion zero mode is localized and what is the width of its wave function [13, 14, 15].

Now let us recall the ordinary four-dimensional free spinor field satisfying the Dirac equation. It is well known that each component of this field satisfies the Klein-Gordon equation, which is a second-order differential equation. Of course, all the components of the spinor field are not independent - with the help of the initial Dirac equation one can restore, for example, the twocomponent spinor $\psi_{R}$ using a solution for the two-component spinor $\psi_{L}$, where the components of $\psi_{L}$ are supposed to be independent and to satisfy the Klein-Gordon equation. The KleinGordon equation is known to have no pathologies, so one can be sure that the whole theory is consistent. The five-dimensional fields $\Psi_{1}$ and $\Psi_{2}$ satisfying equations (22), (23) should be considered as free fields as well, because they are coupled only to the vacuum configurations of the Higgs and gravity fields. Therefore, one expects that in a consistent theory each component of the five-dimensional spinor fields $\Psi_{1}$ and $\Psi_{2}$ (or at least of their linear combinations) also satisfies a five-dimensional second-order differential equation, which contains derivatives in the four-dimensional coordinates only in the form $\square=\eta^{\mu \nu} \partial_{\mu} \partial_{\nu}$, otherwise one may expect the appearance of various pathologies when expanding in Kaluza-Klein modes (which is, in fact, the first step in examining the four-dimensional effective theory), an example of such a pathological behavior will be presented below. The latter is not good taking into account the fact that the components of the five-dimensional spinors make up four-dimensional fermion fields (just like how $\psi_{L}$ and $\psi_{R}$ make up a four-dimensional four-component spinor field), see, for example, [8, 16]. So, let us try to obtain the corresponding second-order differential equations. From (22) and (23) it is not difficult to obtain:

$$
\begin{array}{r}
-\square \Psi_{1}+e^{\sigma}\left(\partial_{5}+2 \sigma^{\prime}\right) e^{\sigma}\left(\partial_{5}+\right. \\
\left.\left.+h \sigma^{\prime}\right) \Psi_{1}+e^{\sigma} \partial_{5}\left(e^{\sigma} F_{1}(z)\right) \gamma^{5} \Psi_{1}-e^{2 \sigma}\left(F_{1}^{2} v(z)\right) \gamma^{5} \Psi_{2}-h e^{2 \sigma} v(z)\left(F_{1}(z)+F_{2}(z)\right) \Psi_{2}(z)\right) \Psi_{1}=0, \\
-\square \Psi_{2}+e^{\sigma}\left(\partial_{5}+2 \sigma^{\prime}\right) e^{\sigma}\left(\partial_{5}+\right. \\
\left.+2 \sigma^{\prime}\right) \Psi_{2}+e^{\sigma} \partial_{5}\left(e^{\sigma} F_{2}(z)\right) \gamma^{5} \Psi_{2}-e^{2 \sigma}\left(F_{2}^{2}(z)+h^{2} v^{2}(z)\right) \Psi_{2} \\
+h e^{\sigma} \partial_{5}\left(e^{\sigma} v(z)\right) \gamma^{5} \Psi_{1}-h e^{2 \sigma} v(z)\left(F_{1}(z)+F_{2}(z)\right) \Psi_{1}=0 .
\end{array}
$$

From (26) and (27) one can see that formally the equations for the components of the fields $\Psi_{1}$ and $\Psi_{2}$ do not decouple. It turns out that in the general case we can not obtain secondorder differential equations for each component of the fields $\Psi_{1}$ and $\Psi_{2}$ (or of their linear combinations) separately, as it happens in the ordinary four-dimensional theory, except several special cases. The first obvious exception is when the following conditions fulfill:

$$
\begin{gathered}
F_{1}(z) \equiv-F_{2}(z), \\
\partial_{5}\left(e^{\sigma} v(z)\right) \equiv 0 .
\end{gathered}
$$

The second condition completely coincides with (16). Introducing the dimensionless coupling constant $\tilde{h}=h \xi$ and taking into account (28) and (29) we can rewrite equations (26) and (27) as

$$
\begin{aligned}
& -\square \Psi_{1}+e^{\sigma}\left(\partial_{5}+2 \sigma^{\prime}\right) e^{\sigma}\left(\partial_{5}+2 \sigma^{\prime}\right) \Psi_{1}+e^{\sigma} \partial_{5}\left(e^{\sigma} F\right) \gamma^{5} \Psi_{1}-\left(e^{2 \sigma} F^{2}+\tilde{h}^{2} \tilde{v}^{2}\right) \Psi_{1}=0, \\
& -\square \Psi_{2}+e^{\sigma}\left(\partial_{5}+2 \sigma^{\prime}\right) e^{\sigma}\left(\partial_{5}+2 \sigma^{\prime}\right) \Psi_{2}-e^{\sigma} \partial_{5}\left(e^{\sigma} F\right) \gamma^{5} \Psi_{2}-\left(e^{2 \sigma} F^{2}+\tilde{h}^{2} \tilde{v}^{2}\right) \Psi_{2}=0
\end{aligned}
$$


where $F(z) \equiv F_{1}(z) \equiv-F_{2}(z)$, which indeed lead to the second-order differential equations for each component of the fields $\Psi_{1}$ and $\Psi_{2}$ separately.

The solution to these equations, corresponding to the zero mode, has the form (it can also be easily obtained from (22), (23) $)$

$$
\begin{aligned}
& \Psi_{1}=C_{f} \exp \left[-\int_{0}^{z} F(y) d y-2 \sigma(z)\right] \psi_{L}(x), \quad i \gamma^{\mu} \partial_{\mu} \psi_{L}-\tilde{h} \tilde{v} \psi_{R}=0, \quad \gamma^{5} \psi_{L}=\psi_{L} \\
& \Psi_{2}=C_{f} \exp \left[-\int_{0}^{z} F(y) d y-2 \sigma(z)\right] \psi_{R}(x), \quad i \gamma^{\mu} \partial_{\mu} \psi_{R}-\tilde{h} \tilde{v} \psi_{L}=0, \quad \gamma^{5} \psi_{R}=-\psi_{R},
\end{aligned}
$$

where again $C_{f}$ is a normalization constant. This solution indeed corresponds to the lowest mode, see Appendix A for details. It is clear that the fields $\psi_{L}$ and $\psi_{R}$ are localized in the vicinity of the same point in the extra dimension. Taken together they make up a four-dimensional Dirac fermion with mass $\tilde{h} \tilde{v}$. As for the physical degrees of freedom corresponding to higher Kaluza-Klein fermion modes, for the case of equations (30), (31) they can be examined exactly in the same way as it was made in [16] for the model with infinite extra dimension.

It should be also mentioned that the fermion action exactly of form (21) with conditions (28) and (29) (but in other notations) was considered in [9] for examining discrete symmetries in brane world models.

It is interesting to note that if the localizing functions $F(z)$ have one and the same form for all fermion fields in the theory (leptons, quarks), then the wave functions of the zero modes also have the same form for different fermions regardless of the four-dimensional mass of the mode (see (32), (33) ). In this case the coupling constants of fermions to gauge bosons in the zero mode sector appear to be exactly the same as in the SM. This happens because the wave functions of all the zero mode gauge bosons do not depend on the coordinate of the extra dimension if relation (29) holds (see Section 2) and all the corresponding vertices (even those containing two different four-dimensional fermions) in fact contain the integral of the same fermion wave function squared, and this integral is equal to unity due to the normalization conditions.

The question arises, whether there are other exceptions in equations (26), (27), leading to second-order differential equations of motion for any form of $v(z)$ ? We found another simple exception for the general case (in principle, there are more exceptions, but they seem to be very unnatural, see Appendix B for a detailed discussion of the decoupling of equations (26), (27)), which is, in fact, rather obvious and follows even from the form of equations (26) Namely, if the relation

$$
F_{1}(z) \equiv F_{2}(z)
$$

is fulfilled, then one can simply add and subtract equations (26), (27) to obtain two independent second-order differential equations for the combinations $\Psi_{1}+\Psi_{2}$ and $\Psi_{1}-\Psi_{2}$, which look like they should not lead to any pathologies.

Relation (34) seems to be rather unphysical (indeed, a consistent localization of the zero modes of fermion fields demands (28), see also, for example, [7, 9, 17]; while it is unclear what could be the physical motivation for the condition $\left.F_{1}(z) \equiv F_{2}(z) \not \equiv 0\right)$; except for the case $F_{1}(z) \equiv F_{2}(z) \equiv 0$, which means that all the fermion fields can freely propagate in the 
bulk. Thus, to examine this case in more detail let us simplify the task and take $\sigma(z) \equiv 0$, $F_{1}(z) \equiv F_{2}(z) \equiv 0$ and $v(z) \not \equiv$ const (for the case $\sigma(z) \equiv 0$ the latter condition does not satisfy $(29))$. The corresponding equations of motion, following from (26) and (27), take the form

$$
\begin{gathered}
-\square\left(\Psi_{1}+\Psi_{2}\right)+\partial_{5}^{2}\left(\Psi_{1}+\Psi_{2}\right)-h^{2} v^{2}\left(\Psi_{1}+\Psi_{2}\right)+h v^{\prime} \gamma^{5}\left(\Psi_{1}+\Psi_{2}\right)=0, \\
-\square\left(\Psi_{1}-\Psi_{2}\right)+\partial_{5}^{2}\left(\Psi_{1}-\Psi_{2}\right)-h^{2} v^{2}\left(\Psi_{1}-\Psi_{2}\right)-h v^{\prime} \gamma^{5}\left(\Psi_{1}-\Psi_{2}\right)=0 .
\end{gathered}
$$

Using these equations it is not difficult to show that, according to the orbifold symmetry conditions (18), (19), the fields $\Psi_{1}$ and $\Psi_{2}$ can be decomposed into the Kaluza-Klein modes as

$$
\begin{aligned}
\Psi_{1} & =\sum_{n}\left(f_{+}^{n}(z) \psi_{L}^{n}(x)-f_{-}^{n}(z) \psi_{R}^{n}(x)\right), \\
\Psi_{2} & =\sum_{n}\left(f_{-}^{n}(z) \psi_{L}^{n}(x)+f_{+}^{n}(z) \psi_{R}^{n}(x)\right),
\end{aligned}
$$

where $\gamma^{5} \psi_{L}=\psi_{L}, \gamma^{5} \psi_{R}=-\psi_{R}$,

$$
f_{+}^{n}(z)=f^{n}(z)+f^{n}(-z), \quad f_{-}^{n}(z)=f^{n}(z)-f^{n}(-z)
$$

and the function $f^{n}(z)$ is a periodic continuously differentiable solution to the equation

$$
m_{n}^{2} f^{n}+\partial_{5}^{2} f^{n}-h^{2} v^{2} f^{n}+h v^{\prime} f^{n}=0
$$

in the interval $[-L, L]$, corresponding to the eigenvalue $m_{n}^{2}$ (recall that $v(-z)=v(z)$ ). According to the general theory [18], the functions $f^{n}(z)$ make up an orthonormal set of eigenfunctions for equation (40), the lowest eigenvalue $m_{0}$ being simple (which means that we get only one fermion with mass $m_{0}$ in the effective four-dimensional theory). Moreover, it is not difficult to show that $m_{0}^{2}>0$ for (40). Thus, the corresponding free theory seems to have no obvious pathologies. But the chiral structure of the zero modes of the fields $\Psi_{1}$ and $\Psi_{2}$ in (37), (38) differs from that in (32), (33). This difference leads to certain problems when taking into account the interactions with the gauge fields. This issue will be discussed in the next section.

What can happen when the decoupling of the equations of motion for the components of the fermion fields seems to be impossible, at least in the standard way (like the one presented in Appendix B)? To show it, let us again simplify the task and consider the case $\sigma(z) \equiv 0$ (the flat five-dimensional space-time). In this case equations (26) and (27) take the form

$$
\begin{gathered}
-\square \Psi_{1}+\partial_{5}^{2} \Psi_{1}+F_{1}^{\prime} \gamma^{5} \Psi_{1}-\left(F_{1}^{2}+h^{2} v^{2}\right) \Psi_{1}+h v^{\prime} \gamma^{5} \Psi_{2}-h v\left(F_{1}+F_{2}\right) \Psi_{2}=0, \\
-\square \Psi_{2}+\partial_{5}^{2} \Psi_{2}+F_{2}^{\prime} \gamma^{5} \Psi_{2}-\left(F_{2}^{2}+h^{2} v^{2}\right) \Psi_{2}+h v^{\prime} \gamma^{5} \Psi_{1}-h v\left(F_{1}+F_{2}\right) \Psi_{1}=0 .
\end{gathered}
$$

The equation for, say, the first component of the field $\Psi_{1}$ can be easily obtained and turns out to be the fourth-order differential equation

$$
\begin{aligned}
{\left[\square-\partial_{5}^{2}-F_{2}^{\prime}+F_{2}^{2}+h^{2} v^{2}\right] \frac{1}{h v\left(F_{1}+F_{2}\right)-h v^{\prime}}\left[\square-\partial_{5}^{2}-F_{1}^{\prime}+F_{1}^{2}+h^{2} v^{2}\right] \Psi_{1}^{(1)} } \\
-\left(h v\left(F_{1}+F_{2}\right)-h v^{\prime}\right) \Psi_{1}^{(1)}=0 .
\end{aligned}
$$

Analogous equations can be obtained for the other components of the field $\Psi_{1}$ and for the components of the field $\Psi_{2}$ (though, as in the four-dimensional case, not all the components 
of the fields $\Psi_{1}$ and $\Psi_{2}$ are completely independent, some of them can be expressed through the other: for example, the field $\Psi_{2}$ can be restored from the field $\Psi_{1}$ with the help of initial equation (22)). It is obvious that even in the flat case $\sigma(z) \equiv 0$ the form of equation (43) poses a question about the possibility of a mathematically consistent isolation of the physical degrees of freedom of the theory. Moreover, since in many cases higher-derivative theories contain pathologies (such as ghosts; see, for example, [19] for details), one may expect pathological behavior in the case under consideration too. On the other hand, usually the systems described by fourth-order equations of motion like the one in (43) have more degrees of freedom than the systems described by second-order equations of motion like the one in (26) with $v(z) \equiv 0$, so in principle one can expect an increase of the number even of the zero modes in addition to other possible pathologies (i.e., one could imagine that there would appear, say, two electrons in the effective four-dimensional theory). We have failed to solve such fourth-order equations of motion analytically even in the simplest cases and we have not an explicit example which could support this statement, however, such a possibility is not excluded by the general reasonings. Thus, in our opinion, one should avoid the appearance of such fourth-order differential equations when constructing multidimensional models, at least to be sure that the resulting theory is devoid of any pathologies and the physical degrees of freedom can be isolated in a mathematically consistent way using the well-developed theory of second-order differential equations.

An important comment is in order here. In many brane world models the expansion in the Kaluza-Klein modes for the fields $\Psi_{1}$ and $\Psi_{2}$ is performed without taking into account the interaction with the Higgs field (indeed, in the case $v(z) \equiv 0$ the corresponding differential equations are indeed second-order and one does not expect any pathologies). When the interaction with the Higgs field is taken back into account, all the off-diagonal entries of the corresponding (infinite) mass matrix are, in the general case, nonzero, which is not an unexpected result, because the orthogonality conditions for the case $v(z) \equiv 0$ are not valid for the case $v(z) \not \equiv 0$, $v(z) \not \equiv \tilde{v} e^{-\sigma}$ (including the generalized functions like the delta-function). It is clear that, in principle, the resulting fields do not represent the physical degrees of freedom of the theory and cannot be used for consistent calculations, which poses the question about the diagonalization of the mass matrix in the effective four-dimensional theory by algebraic (maybe perturbative) methods. However, as it was noted above, in the general case the systems described by fourthorder equations of motion like the one in (43) have more degrees of freedom than the systems described by second-order equations of motion like the one in (26) with $v(z) \equiv 0$. The latter makes the use of the perturbation theory and the subsequent diagonalization of the mass matrix questionable, because in the general case this mass matrix, obtained using the solutions of equations (26) and (27) with $v(z) \equiv 0$, may not include all the degrees of freedom (including possible pathological modes) described by equations (26) and (27) with $v(z) \not \equiv 0$. We note that it is a nonperturbative effect, which may appear no matter what is the relation between the energy scale of the Higgs field and the typical energy scale of five-dimensional theory 1 In some sense this situation is similar to the case of $U(1)$ massless gauge field: if one adds the mass term to the action, the third degree of freedom arises, no matter how small the mass of

\footnotetext{
${ }^{1}$ There is a simple algebraic example, demonstrating an analogous nonperturbative effect. The equation $x-1=0$ has only one real root, whereas the equation $\alpha x^{2}+x-1=0$ with $|\alpha| \ll 1$ has two real roots. The first root $x \approx 1-\alpha$ indeed can be obtained perturbatively, whereas it is not so for the other root $x \approx-\frac{1}{\alpha}-1+\alpha$.
} 
such a "photon" is. It is impossible to trace such an effect by first performing the KK decomposition in the vacuum with $v(z) \equiv 0$ and only then considering the interaction with the nonzero vacuum solution of the Higgs field. For this reason we think that the only consistent way of deriving an effective four-dimensional action in brane world models is to consider first the vacuum solution for the Higgs field (and for other fields with nonzero vacuum solutions, if they exist) and only then to perform the Kaluza-Klein decomposition (if it is possible) checking the absence of pathologies at least in the free theory.

\section{Interactions in the effective theory}

To demonstrate in a simple way, how possible latent problems can pop up in the four-dimensional effective theory, corresponding to equations (35) and (36), let us consider a fivedimensional action, describing fermion fields minimally coupled to the $S U(2) \times U(1)$ gauge fields in the flat $(\sigma(z) \equiv 0)$ space-time:

$$
S=\int d^{4} x d z\left(i \overline{\hat{\Psi}}_{1} \Gamma^{M} D_{M} \hat{\Psi}_{1}+i \bar{\Psi}_{2} \Gamma^{M} D_{M} \Psi_{2}-\sqrt{2} h\left[\left(\overline{\hat{\Psi}}_{1} H\right) \Psi_{2}+\text { h.c. }\right]\right) .
$$

Here the $S U(2)$ doublet, constructed from five-dimensional spinors, is denoted by

$$
\hat{\Psi}_{1}=\left(\begin{array}{c}
\Psi_{1}^{\nu} \\
\Psi_{1}^{\psi}
\end{array}\right), \quad \overline{\hat{\Psi}}_{1}=\left(\bar{\Psi}_{1}^{\nu}, \bar{\Psi}_{1}^{\psi}\right)
$$

and the five-dimensional $S U(2)$ singlet is denoted by $\Psi_{2}$. The covariant derivatives are defined by

$$
\begin{aligned}
D_{M} \hat{\Psi}_{1} & =\left(\partial_{M}-i g \frac{\tau^{a}}{2} A_{M}^{a}+i \frac{g^{\prime}}{2} B_{M}\right) \hat{\Psi}_{1}, \\
D_{M} \Psi_{2} & =\left(\partial_{M}+i g^{\prime} B_{M}\right) \Psi_{2} .
\end{aligned}
$$

The vacuum solution for the Higgs field is supposed to have the form

$$
H_{0} \equiv\left(\begin{array}{c}
0 \\
\frac{v(z)}{\sqrt{2}}
\end{array}\right)
$$

with $v(z) \not \equiv$ const.

First, let us consider the free theory. We will be interested only in the lowest mode sector, so we neglect all the higher Kaluza-Klein modes of gauge and fermion fields. According to (35) -(40), we can represent the fermion zero modes as (below we will omit the superscript "0" for the zero $(n=0)$ modes of the fields)

$$
\hat{\Psi}_{1}(x, z)=\left(\begin{array}{l}
\xi \nu_{L}(x) \\
f_{+}(z) \psi_{L}(x)-f_{-}(z) \psi_{R}(x)
\end{array}\right), \quad \Psi_{2}(x, z)=f_{-}(z) \psi_{L}(x)+f_{+}(z) \psi_{R}(x),
$$

where $\nu_{L}=\gamma^{5} \nu_{L}$. The factor $\xi=\frac{1}{\sqrt{2 L}}$ is just the canonically normalized wave function of the zero mode of the field $\Psi_{1}^{\nu}$ (since this field does not interact with the vacuum solution of 
the Higgs field $H$, in the flat background and with $F_{1}(z) \equiv F_{2}(z) \equiv 0$ its wave function is a constant). Substituting (49) into (44), dropping the terms with gauge fields and integrating over the coordinate of the extra dimension, one can obtain the standard four-dimensional action for the free fermion fields 2

$$
S=\int d^{4} x\left(i \overline{\nu_{L}} \gamma^{\mu} \partial_{\mu} \nu_{L}+i \bar{\psi} \gamma^{\mu} \partial_{\mu} \psi-m \bar{\psi} \psi\right)
$$

where $\psi=\psi_{L}+\psi_{R}$. In order to have the canonically normalized kinetic term of the field $\psi$ in (50), the condition

$$
a^{2}+b^{2}=1, \quad a^{2}=\int d z f_{+}^{2}(z), \quad b^{2}=\int d z f_{-}^{2}(z) .
$$

must be fulfilled. The mass $m$ is the eigenvalue of the problem (40), corresponding to the eigenfunction $f(z)$. By tuning the coupling constant $h$ one can, in principle, get the desired value of the mass $m$.

Now let us turn to examining the interactions with the gauge bosons. In order to isolate the effects caused only by the fermions, below we choose the following ansatz for the zero modes of the gauge fields:

$$
A_{\mu}^{a}(x, z) \equiv A_{\mu}^{a}(x), \quad B_{\mu}(x, z) \equiv B_{\mu}(x)
$$

for simplicity we will also drop the components $A_{5}^{a}(x, z)$ and $B_{5}(x, z)$ of these fields. We do not discuss here possible ways for obtaining this ansatz in a consistent way. For example, one may simply imagine that there exists a second Higgs field, interacting with the gauge fields only, which provides the necessary forms of the corresponding wave functions.

Passing to the physical degrees of freedom (11), using (49), (52) and integrating over the coordinate of the extra dimension, we can obtain the effective four-dimensional action, describing the interaction of the zero mode fermions with the gauge bosons. We do not present the explicit calculations here, they are straightforward. It is not difficult to show that the electromagnetic coupling constant appears to be the same as in the SM (from here and below "the same as in the SM" means that it can be expressed through the constants $g$ and $g^{\prime}$ exactly in the same way as it happens in the SM). The coupling constant of the interaction with the charged gauge bosons is found to be

$$
g \frac{1}{\sqrt{2 L}} \int d z f_{+}(z)
$$

instead of $g$ in the SM (recall that the wave function, corresponding to the field $\nu_{L}$, is just $\frac{1}{\sqrt{2 L}}$ ). As for the interaction of the field $\psi$ with the neutral gauge boson $Z$, the vector coupling constant appears to be the same as in the SM, whereas the axial coupling constant has the form

$$
g_{A}=g_{A}^{S M}\left(a^{2}-b^{2}\right) .
$$

It is clear that in the general case $c=\frac{1}{\sqrt{2 L}} \int d z f_{+}(z) \neq 1$ and, according to (51), $a^{2}-b^{2} \neq 1$. Meanwhile, in order to get rid of the difference with the well known parameters of the SM,

\footnotetext{
${ }^{2}$ In the derivation of action (50) it is convenient to use the equations $f_{+}^{\prime}+h v f_{-}=-m f_{-}$, $f_{-}^{\prime}+h v f_{+}=m f_{+}$, which are fulfilled if equation (40) holds.
} 
one should have $c=1$ and $a^{2}-b^{2}=1$ (or the values which are close to unity with a good accuracy). The latter can be achieved if $b=0$, which means that $f(z) \equiv$ const; in this case $c=1$ too. But, according to (40), the condition $f(z) \equiv$ const means that $v(z) \equiv$ const, which again corresponds to (29) with $\sigma(z) \equiv 0$. Thus, the farther $v(z)$ from a constant is, the farther the values of the corresponding coupling constants are from those of the SM.

It is obvious that for the non-flat case $\sigma(z) \not \equiv 0$ with $\partial_{5}\left(e^{\sigma} v(z)\right) \not \equiv 0$ the problems, completely analogous to those described above, are also expected to arise in the four-dimensional effective theory, though the calculations appear to be much more bulky than in the case $\sigma(z) \equiv 0$. The only difference in the warped case is that now the farther $v(z)$ from $\sim e^{-\sigma}$ is, the farther the values of the corresponding coupling constants are from those of the $\mathrm{SM} 3$ (note that in a realistic theory with $\partial_{5}\left(e^{\sigma} v(z)\right) \not \equiv 0$ the shapes of the zero mode gauge boson wave functions also differ from a constant, which provides additional deviations from the standard values of the SM parameters like in [4, 5]). For example, the natural choice $v(z) \equiv$ const may lead, in principle, either to an unacceptable theory or put it out of the reach of the present day experiments.

As a final remark to this section, let us draw an analogy with the case of gauge fields. As it was mentioned in Section 2, the modification of the shapes of the gauge boson wave functions due to the interaction with the vacuum solution of the Higgs field may affect the fourdimensional effective theory considerably [4, 5]. In the most cases the interaction of fermion fields with the vacuum solution of the Higgs field affects the corresponding effective theory either in an analogous way (by a modification of the zero mode wave functions and the chiral structure), or even more dramatically, leading to pathologies like those discussed in Section 3. The latter clearly indicates that the interaction of fermion fields with the vacuum solution of the Higgs field should be treated much more carefully than it is usually done.

\section{Conclusion and final remarks}

As it was demonstrated in the previous sections, the only obvious possibility to automatically get a self-consistent, from the theoretical point of view, four-dimensional SM in a fivedimensional brane world model (i.e., without possible pathologies in the free theory and with the correct couplings) is to have a vacuum solution of form (16) for the Higgs field together with (28). Condition (28) corresponds, in fact, both to the case of localized fermion zero modes and to the case when the fermion fields can freely propagate in the bulk (if $\left.F_{1}(z) \equiv F_{2}(z) \equiv 0\right)$. 4 It is not difficult to check that in this case the zero mode fermion and gauge boson sectors of the resulting effective theory indeed exactly reproduce those of the SM, including the interactions, at least for the case of the standard form of five-dimensional gauge invariant action (of course, if the localizing functions $F(z)$ are one and the same for all the fields, corresponding to different

\footnotetext{
${ }^{3}$ This reasoning is valid only for the case $F_{1}(z) \equiv F_{2}(z) \equiv 0$. If $F_{1}(z) \equiv F_{2}(z) \not \equiv 0$, there should be deviations of the coupling constants from those of the SM even for $\partial_{5}\left(e^{\sigma} v(z)\right) \equiv 0$ due to the nonzero rotation angle $\theta$, see Appendix B.

${ }^{4}$ As it was shown in Appendix B, there may exist other exceptions in equations (26), (27), leading to secondorder differential equations of motion. But, according to the results presented above, it is improbable that such unnatural cases could lead to a completely acceptable effective theory.
} 
four-dimensional SM particles). The corresponding calculations are straightforward and we do not present them here. The self-consistency and automatic conformity to the SM of the zero mode fermion and gauge boson sectors of the resulting four-dimensional effective theory for (28) and (29), follow from the fact that only in this case equations (26) and (27) for the components of the five-dimensional fermions $\Psi_{1}$ and $\Psi_{2}$ decouple, which provides the correct chiral structure of the corresponding lowest Kaluza-Klein modes. Deviations from these conditions may lead either to pathologies or to a variance between the resulting zero mode four-dimensional effective theory and the SM. The latter may result in severe constraints on the parameters of five-dimensional theory and put it, in principle, out of the reach of the present day experiments in full analogy with how in happens in the gauge boson sector [4, 5].

It should be noted that the exponential profile of the vacuum solution for the Higgs field, leading to its localization near the TeV brane in the Randall-Sundrum model [2], was discussed earlier [15]. Our results demonstrate that in order to have a possibility to localize the zero modes of fermion fields in a consistent way (using an appropriate form of the function $F(z)$ in (30) and (31)), the profile of the vacuum solution of the Higgs field should have exactly the form (16).

Unfortunately, the restriction on the vacuum profile of the Higgs field poses several problems for the case of localized fermion zero modes (i.e., when $F(z) \not \equiv 0$ ), which should be necessarily addressed. First, the profile of the vacuum solution for the Higgs field appears to be strongly related to the form of the warp factor of the model. This demands an extra fine-tuning for the scalar field potential. Indeed, even for the simplest case of the Randall-Sundrum setup [2] with $\sigma(z)=-k|z|$ and without taking into account the backreaction of the Higgs field on the background metric, the fine-tuned bulk Higgs potential should have the form

$$
V\left(H^{\dagger} H\right)=-3 k^{2} H^{\dagger} H
$$

to get the vacuum solution (16). Of course, one should also add fine-tuned brane-localized potentials, including a term specifying the value of the constant $\tilde{v}$ (at least on one of the branes). If one takes more realistic cases of stabilized models, in which the warp factors have a more complicated form (like the one in [20]), the form of the Higgs scalar field potential appears to be such that it can not be represented in an analytical form. Of course, such a situation looks unnatural, at least in the absence of a symmetry that can support such a fine-tuning of the Higgs potential. Moreover, backreaction of the Higgs field affects the background metric, whereas quantum corrections modify the scalar field potential and, consequently, the vacuum solution for the Higgs field. Both effects lead to breakdown of the fine-tuned relation between the warp factor and the vacuum solution for the Higgs field.

Second, in a scenario with localized zero modes of fermion fields the Higgs field and the stabilizing scalar field cannot be unified, as it was proposed in [21]. Indeed, a consistent stabilization mechanism (like the one proposed in [20]) is based on fixing the values of the stabilizing scalar fields on the branes (at the points $z=0$ and $z=L$ ). On the other hand, warped brane world models are interesting if the function $e^{2 \sigma}$ has exponentially different values on the branes. The latter means that by taking the Higgs field as the stabilizing field in such a theory, one introduces a new hierarchy into the model (because $v(0) \ll v(L)$ ). For example, the RandallSundrum model [2] was proposed to solve the hierarchy problem of gravitational interaction, so 
it also looks unnatural to add an extra hierarchy into such a model. Moreover, in order to get a massive radion, one should take into account the backreaction of the stabilizing field on the background metric [20]. But if the stabilizing field is the Higgs field with vacuum solution (16), then the range of allowed scalar field potentials and warp factors narrows considerably (which clearly follows from the self-consistent system of equations for the background configuration of the metric and the stabilizing scalar field, which can be found in [20]).

One may suppose that if at least the fermion fields are located exactly on the brane, then the Higgs field can also be located on the brane, which looks as a solution to the problem (of course, if we do not take into account the gauge fields, see [4]). However, the only realistic field-theoretical mechanism of fermion localization, which can be used for calculations, is based on the idea that initially the fermion fields propagate in the whole five-dimensional space-time, whereas only the lowest modes appear to be localized on the brane due to an interaction with some defect (for example, with a domain wall like in [11]), see the discussion in Section 3. This is exactly the situation, which is realized in equations (32), (33), so one can take an appropriate form of the function $F(z)$ to make the width of the wave function of the localized fermion as small as necessary. Meanwhile, the profile of the Higgs field does not depend on the form of the function $F(z)$, so even for an extremely narrow wave function of a localized mode (which taken squared can be even approximated by the delta-function for calculations) the "right" profile of the Higgs field, which does not lead to fourth-order differential equations, should still have the form (16). The latter poses a question whether there exists a field-theoretical mechanism of fermion localization, leaving more freedom for the choice of a vacuum profile of the Higgs field in the extra dimension.

It should be noted that the only obvious exception is the model with the flat five-dimensional background metric like the one proposed in [3], for which the four-dimensional SM can be constructed from a five-dimensional theory without unnatural fine-tunings and restrictions. In such a case the vacuum solution for the Higgs field must be just a constant, which admits a variety of scalar field potentials including the standard Higgs potential (but leaving unsolved the problem of the stabilization of the extra dimension size).

\section{Acknowledgements}

The authors are grateful to E. Boos and S. Keizerov for discussions and useful remarks. The work was supported by grant 14-12-00363 of Russian Science Foundation.

\section{Appendix A: Mass of the lowest localized fermion mode}

Let us take equation (30) and substitute $\Psi_{1}(x, z)=\psi_{L}(x) f_{L}(z)$ with $\square \psi_{L}+\mu^{2} \psi_{L}=0$ and $\gamma^{5} \psi_{L}=\psi_{L}$ into it. We get

$$
\left(\mu^{2}-\tilde{h}^{2} \tilde{v}^{2}\right) f_{L}+e^{\sigma}\left(\partial_{5}+2 \sigma^{\prime}\right) e^{\sigma}\left(\partial_{5}+2 \sigma^{\prime}\right) f_{L}+e^{\sigma} \partial_{5}\left(e^{\sigma} F\right) f_{L}-e^{2 \sigma} F^{2} f_{L}=0 .
$$


Multiplying this equation by $e^{3 \sigma} f_{L}$, integrating over the coordinate of the extra dimension $z$ and performing integration by parts in two terms, we arrive to the following equality:

$$
\left(\mu^{2}-\tilde{h}^{2} \tilde{v}^{2}\right) \int d z e^{3 \sigma} f_{L}^{2}=\int d z e^{5 \sigma}\left(f_{L}^{\prime}+2 \sigma^{\prime} f_{L}+F f_{L}\right)^{2}
$$

Since both integrals are nonnegative, we get $\mu^{2}-\tilde{h}^{2} \tilde{v}^{2} \geq 0$, which means that the lowest mode indeed has mass $\tilde{h} \tilde{v}$. A completely analogous procedure can be performed for the other substitution $\Psi_{1}(x, z)=\psi_{R}(x) f_{R}(z)$ with $\square \psi_{R}+\mu^{2} \psi_{R}=0$ and $\gamma^{5} \psi_{R}=-\psi_{R}$, as well as for the field $\Psi_{2}$.

\section{Appendix B: Decoupling of the equations of motion for fermions}

Let us consider equations (26) and (27) for the left-handed fermions such that $\Psi_{1}^{L}=\gamma^{5} \Psi_{1}^{L}$, $\Psi_{2}^{L}=\gamma^{5} \Psi_{2}^{L}$. These equations can be rewritten in the matrix form as

$$
\hat{L}(x, z)\left(\begin{array}{c}
\Psi_{1}^{L} \\
\Psi_{2}^{L}
\end{array}\right)+\hat{M}(z)\left(\begin{array}{c}
\Psi_{1}^{L} \\
\Psi_{2}^{L}
\end{array}\right)=0
$$

where $\hat{L}(x, z)$ is a diagonal operator with equal diagonal elements, which includes derivatives, and the matrix $\hat{M}(z)$ looks like

$$
\hat{M}(z)=\left(\begin{array}{cc}
q_{1}(z) & p(z) \\
p(z) & q_{2}(z)
\end{array}\right)
$$

with

$$
\begin{aligned}
q_{1}(z) & =e^{\sigma} \partial_{5}\left(e^{\sigma} F_{1}(z)\right)-e^{2 \sigma} F_{1}^{2}(z), \\
q_{2}(z) & =e^{\sigma} \partial_{5}\left(e^{\sigma} F_{2}(z)\right)-e^{2 \sigma} F_{2}^{2}(z), \\
p(z) & =h e^{\sigma} \partial_{5}\left(e^{\sigma} v(z)\right)-h e^{2 \sigma} v(z)\left(F_{1}(z)+F_{2}(z)\right) .
\end{aligned}
$$

The form of equation (57) suggests that the decoupling of the equations of motion for the fermion fields is equivalent to the diagonalization of the matrix $\hat{M}(z)$, so the question is how to diagonalize the matrix $\hat{M}(z)$ except for the obvious case $p(z) \equiv 0$. Since this matrix is symmetric, it can be diagonalized with the help of a rotation matrix $\hat{R}$ such that

$$
\hat{R}^{T} \hat{M} \hat{R}=\operatorname{diag}\left(\lambda_{1}, \lambda_{2}\right), \quad \hat{R}=\left(\begin{array}{cc}
\cos \theta & -\sin \theta \\
\sin \theta & \cos \theta
\end{array}\right) .
$$

The eigenvalues of the matrix $\hat{M}(z)$ can be easily found by the standard procedure and take the form

$$
\lambda_{1,2}(z)=\frac{q_{1}+q_{2} \pm \sqrt{\left(q_{1}-q_{2}\right)^{2}+4 p^{2}}}{2}
$$

A very important point is that the rotation angle $\theta$ should not depend on the coordinate of the extra dimension $z$, otherwise the rotation matrix $\hat{R}$ would not pass through the operator $\hat{L}$, 
which contains derivatives. Since it is the eigenvectors of the matrix $\hat{M}$ that form the rotation matrix $\hat{R}$, it is necessary to find conditions under which these eigenvectors do not depend on the coordinate of the extra dimension. From the equation, say, for the first eigenvalue and eigenvector

$$
\hat{M}(z)\left(\begin{array}{c}
\cos \theta \\
\sin \theta
\end{array}\right)=\lambda_{1}(z)\left(\begin{array}{c}
\cos \theta \\
\sin \theta
\end{array}\right)
$$

we can easily get

$$
\tan \theta=\frac{q_{2}-q_{1}+\sqrt{\left(q_{1}-q_{2}\right)^{2}+4 p^{2}}}{2 p} .
$$

It is clear that in the general case the angle $\theta$ depends on the coordinate of the extra dimension. An obvious exception is in general $q_{1}(z) \equiv q_{2}(z)$.

Thus, according to the results, presented above, in the general case we have two conditions for the left-handed fermions, for which the mixing matrix $\hat{M}$ is either diagonal or can be diagonalized in the standard way. They are

$$
\begin{aligned}
\partial_{5}\left(e^{\sigma} v\right) & =e^{\sigma} v\left(F_{1}+F_{2}\right), \\
\partial_{5}\left(e^{\sigma}\left(F_{1}-F_{2}\right)\right) & =e^{\sigma}\left(F_{1}^{2}-F_{2}^{2}\right) .
\end{aligned}
$$

The procedure, completely analogous to the one presenter above, can be also performed for the right-handed fermions $\left(\Psi_{1}^{R}=-\gamma^{5} \Psi_{1}^{R}, \Psi_{2}^{R}=-\gamma^{5} \Psi_{2}^{R}\right)$, leading to

$$
\begin{aligned}
\partial_{5}\left(e^{\sigma} v\right) & =-e^{\sigma} v\left(F_{1}+F_{2}\right), \\
\partial_{5}\left(e^{\sigma}\left(F_{1}-F_{2}\right)\right) & =-e^{\sigma}\left(F_{1}^{2}-F_{2}^{2}\right) .
\end{aligned}
$$

The most general and simple model-independent conditions, following from (666)-(69), are just (28), (29) or (34), the latter case giving $\theta= \pm \frac{\pi}{4}$, which corresponds to the combinations of the fields in (35), (36). Of course, in principle one may consider other combinations of conditions (66) -(69): (66) together with (69) or (67) together with (68); or there may exist other specific choices of the functions $F_{1}(z), F_{2}(z)$ and $v(z)$ leaving the rotation angle $\theta$ independent on the coordinate of the extra dimension. But such cases seem to be much more unnatural, while they also lead to relations between the functions $F_{1}(z), F_{1}(z), v(z)$ and $\sigma(z)$.

\section{References}

[1] N. Arkani-Hamed, S. Dimopoulos and G. R. Dvali, Phys. Lett. B 429 (1998) 263.

[2] L. Randall and R. Sundrum, Phys. Rev. Lett. 83 (1999) 3370.

[3] T. Appelquist, H.C. Cheng and B.A. Dobrescu, Phys. Rev. D 64 (2001) 035002.

[4] C. Csaki, J. Erlich and J. Terning, Phys. Rev. D 66 (2002) 064021.

[5] G. Burdman, Phys. Rev. D 66 (2002) 076003.

[6] V.A. Rubakov, Phys. Usp. 44 (2001) 871. 
[7] S.L. Dubovsky, V.A. Rubakov and P.G. Tinyakov, Phys. Rev. D 62 (2000) 105011.

[8] C. Macesanu, Int. J. Mod. Phys. A 21 (2006) 2259.

[9] R. Casadio and A. Gruppuso, Phys. Rev. D 64 (2001) 025020.

[10] S. Chang, J. Hisano, H. Nakano, N. Okada and M. Yamaguchi, Phys. Rev. D 62 (2000) 084025 .

[11] V.A. Rubakov and M.E. Shaposhnikov, Phys. Lett. B 125 (1983) 136.

[12] N. Arkani-Hamed and M. Schmaltz, Phys. Rev. D 61 (2000) 033005.

[13] Y. Grossman and M. Neubert, Phys. Lett. B 474 (2000) 361.

[14] T. Gherghetta and A. Pomarol, Nucl. Phys. B 586 (2000) 141.

[15] S.J. Huber and Q. Shafi, Phys. Lett. B 498 (2001) 256.

[16] M.N. Smolyakov, Phys. Rev. D 85 (2012) 045036.

[17] A.A. Andrianov, V.A. Andrianov, P. Giacconi and R. Soldati, JHEP 0307 (2003) 063.

[18] E.A. Coddington and N. Levinson, "Theory of Ordinary Differential Equations", McGrawHill, New York, 1955.

[19] R.P. Woodard, Lect. Notes Phys. 720 (2007) 403.

[20] O. DeWolfe, D.Z. Freedman, S.S. Gubser and A. Karch, Phys. Rev. D 62 (2000) 046008.

[21] M. Geller, S. Bar-Shalom and A. Soni, Phys. Rev. D 89 (2014) 095015. 